\title{
An investigation of temporal adaptive solution of Richards' equation for sharp front problems
}

\author{
M Sayful Islam ${ }^{1}$, M khayrul Hasan ${ }^{2}$ \\ ${ }^{I}$ Dept. of Mathematics, Shahjalal University of Science \& Technology, Sylhet-3114, Bangladesh \\ ${ }^{2}$ Dept. of Mathematics, Shahjalal University of Science \& Technology, Sylhet-3114, Bangladesh
}

\begin{abstract}
Accurate, reliable, efficient, and robust simulation of groundwater flow in the unsaturated zone for the problems that characterized by sharp fronts in both space and time is computationally expensive. The accurate numerical solution of these problems by standard approaches with uniform spatial and temporal discretization usually inefficient and simulation is too costly. Moreover, it is very difficult to obtain explicit solution of Richards' equation by using standard time integration unless very small time steps are used in the integration process. Economical and robust solution may be achieved with variable time step size instead of constant time step size use. In this study, adaptive method in time is used to solve Richards' equation with finite difference technique. Temporal adaptation is accomplished by using variable order, variable step size approximation. We show how a differential algebraic equation can give accurate solution, have good mass balance properties and more economical for a wide range solution accuracy. The accuracy and computational efficiency of the method are evaluated by comparison with a uniform spatial discretization that is adaptive in time for three problems simulating one-dimensional flow processes in unsaturated porous media. The results indicate that the method is quite competitive with spatially and temporally adaptive approach. We conclude that the method can be effectively implemented and efficient alternative to standard approaches for simulating variably saturated flow in one spatial dimension.
\end{abstract}

Keywords: Variably saturated flow, Finite difference, Numerical solution, Richards' equation, Method of lines

\section{Introduction}

In recent years, the attention of groundwater simulation has been increased, because of growing the industrial, municipal and agricultural activities that the quality of subsurface environment is being unfavourably affected by them. Fluid flow in unsaturated porous media is governed by Richards' equation (RE) which is closed by the constitutive relations to describe the relationship among the fluid pressures, saturations, and relative permeabilities[1, 2]. It is highly non-linear parabolic partial differential equation (PDE) which is often difficult to approximate since it does not have a closed-form analytical solution.

To obtain the numerical solution of RE, it is needed to solve the suitable form of the equation, the closed form of the constitutive relations are used, the spatial approximation, the temporal approximation, the nonlinear equation solution, and the linear equation solution methods. For each of these facts standard approaches have evolved, while latest advancements recommend potentially attractive alternatives to the standard choices in some cases [3].

It is suitable to decouple the issues of temporal and spatial accuracy for solving RE numerically. Loworder finite difference or finite element spatial approximations and low-order time integration techniques are the most common approaches to approximate the RE $[4,5]$. Moreover, currently, most of the variably saturated flow simulations are done based upon fixed spatial grids and either fixed time-step or an empirically based adaptive time stepping method [6,7]. The numerical stability of the finite element model is enhanced by mass lumping since earlier findings indicate that reliable mass formulation might cause numerical oscillations $[6,8$, 9]. Previous studies emphasize the importance of appropriate treatment of the time derivative for consistent numerical simulations $[6,10]$. The backward Euler and Crank-Nicolson schemes are the commonly used time stepping schemes. In addition, the three-level Lees' scheme, the Douglas-Jones predictor corrector method, implicit Runge-Kutta scheme and backward difference scheme are used to approximate the RE [11, 12].

It is require that the subject of significant research studies to the solution of the non-linear algebraic systems that arise in implicit numerical discretizations of RE. To resolve the nonlinearities, there are some iterative schemes have been proposed [10, 13, 14], e.g., Picard and Newton iteration methods, fast secant, and relaxation methods as well as non-iterative methods (e.g., the implicit factored scheme). In practice, the Picard method is prevalent due to its simple formulation and satisfactory performance [15].

Regardless of the above mentioned, for the certain class of difficult test problems, especially those that give rise to sharp fronts that propagate through the problem domain have significant issues of robustness and efficiency [3]. These sharp fronts in both space and time can require key changes in spatial adjustment as a function of temporal evolution of the problem. Numerical methods for RE were mostly restricted to 
straightforward time stepping schemes together with finite difference or finite element spatial approximations. Fixed time step and heuristic are the two commonly used simple time stepping strategies, and these are crude and wasteful of computational resources within hydrological simulations [11]. Theoretically, the mixed formulation of RE suggest the fastest possible convergence rates. On the other hand, the difficulty of data structures for some combined adaptive methods can be substantial [16].

The solution of RE based on differential algebraic equations (DAE)/method of lines (MOL) approaches are more robust and efficient to traditional fixed and adaptive time-step approaches for solving difficult sharpfront problems [17]. Temporal truncation error evaluation were used explicitly to control the solution order, which ranged from first to fifth order in time-step size in this method. Time adaptation integration methods with variable-step variable-order (up to fifth) DAE solver (DASPK) integrators $[3,17]$ and lower order adaptive backward Euler and related schemes $[10,12]$ have been developed. For the fixed and heuristic time stepping schemes, accuracy and efficiency are gained significantly with formal truncation error control and also improves the mass balance based on the $\psi$ formulation of RE. Hence, unconditional stability is an essential property of an effective time stepping scheme for RE, due to the stiffness of spatially discrete parabolic PDE [10].

This paper begins with a presentation of the RE, including a description of the numerical model used to discretize the spatial terms. Also the constitutive relationship, MOL, measures of performance and MATLAB ODE solvers are discussed in section 2. Three numerical tests are illustrated in section 3 to emphasize efficiency and robustness of the proposed method. In section 4 we evaluate the accuracy of the scheme. In addition to obtain accuracy and efficiency, we also discuss briefly other features of the method including root mean square error, modeling efficiency and mass balance errors. In the last section, we conclude our final remarks.

\subsection{Problem formulation}

\section{Numerical Procedures}

RE may be formulated in several forms, depending on whether pressure ( $\psi$-based form), moisture ( $\theta$-based form), or both (mixed form) are used as state variables. The one dimensional pressure-head form of RE is given by:

$$
\left[c(\psi)+S_{s}\left(\frac{\theta}{\theta_{s}}\right)\right] \frac{\partial \psi}{\partial t}=\frac{\partial}{\partial z}\left[K(\psi)\left(\frac{\partial \psi}{\partial z}+1\right)\right]
$$

where $\psi$ is the pressure head $[\mathrm{L}], \theta(\psi)$ is the volumetric soil moisture content $\left[\mathrm{L}^{3} \mathrm{~L}^{-3}\right], \mathrm{K}(\psi)$ is the nonnegative hydraulic conductivity $\left[\mathrm{LT}^{-1}\right], \mathrm{t}$ is the time $[\mathrm{T}]$, and $\mathrm{z}$ is the vertical coordinate assumed positive upward [L], $c(\psi)=\frac{d \theta}{d \psi}$ is the moisture capacity $\left[\mathrm{L}^{-1}\right]$, and $\mathrm{S}_{\mathrm{s}}$ is the specific storage coefficient, which accounts for fluid matrix compressibility. In accounting for the effects of specific storage, the governing differential equation is an extension of the classical RE.

The $\psi$-based form of RE is the most commonly used numerical approach because it can be applied to both saturated and unsaturated conditions and of compliant heterogeneous soils. Conversely, very poor preservation of mass balance problems, undesirable time-step limitations [9] and comparatively slow convergence [18] are shown which imply seriously undermines its physical basis [19].

The equation (|ref $\{$ eqm:1 $\}$ ) can be rewrite in the form:

$$
\frac{\partial \psi}{\partial t}=\frac{1}{c(\psi)+S_{S}\left(\frac{\theta}{\theta_{S}}\right)} \frac{\partial}{\partial z}\left[K(\psi)\left(\frac{\partial \psi}{\partial z}+1\right)\right],
$$

or,

where $S_{a}(\psi)=\frac{\theta(\psi)}{\theta_{s}}$.

$$
\frac{\partial \psi}{\partial t}=\frac{1}{c(\psi)+S_{S} S_{a}(\psi)} \frac{\partial}{\partial z}\left[K(\psi)\left(\frac{\partial \psi}{\partial z}+1\right)\right]
$$

To complete this RE model, we need to consider auxiliary conditions of the form:

$$
\begin{aligned}
& \psi(z, t=0)=\psi_{0}(z), \\
& \psi(z=0, t>0)=\psi_{1}, \\
& \psi(z=Z, t>0)=\psi_{2}
\end{aligned}
$$

where $\mathrm{Z}$ is the length of the domain and $\psi_{0}$ (defined as initial condition) may be a function of space, but $\psi_{1}$ (bottom boundary condition) and $\psi_{2}$ (top boundary condition) are constants. These simple conditions are adequate to develop a meaningful set of test problems.

\subsection{Constitutive relationship}

Solution of RE requires constitutive relations between the dependent variable (pressure head) and the nonlinear terms (moisture content, moisture capacity, and conductivity). The soil characteristic curves used in the test cases are given the van Genuchten model [2] which is the most commonly employed model and is given by: 


$$
\begin{aligned}
& \theta(\psi)=\theta_{r}+\frac{\theta_{S}-\theta_{r}}{\left[1+|\alpha \psi|^{n}\right]^{m}}, \quad \text { if } \quad \psi \leq 0 \\
& \theta(\psi)=\theta_{s}, \quad \text { if } \quad \psi>0 \\
& K(\psi)=K_{s}\left[\frac{\theta-\theta_{r}}{\theta_{s}-\theta_{r}}\right]^{\frac{1}{2}}\left\{1-\left[1-\left(\frac{\theta-\theta_{r}}{\theta_{s}-\theta_{r}}\right)^{\frac{1}{m}}\right]^{m}\right\}^{2}, \quad \text { if } \quad \psi \leq 0 \\
& K(\psi)=K_{s}, \quad \text { if } \quad \psi>0 \\
& c(\psi)=\alpha m n \frac{\theta_{s}-\theta_{r}}{\left[1+|\alpha \psi|^{n}\right]^{m+1}}|\alpha \psi|^{n-1}, \quad \text { if } \quad \psi \leq 0 \\
& c(\psi)=0, \quad \text { if } \quad \psi>0
\end{aligned}
$$

where $\theta_{r}$ is the residual volumetric water content, $\theta_{s}$ is the porosity, $\alpha$ is the mean pore size, and $n=1-1 / m$ is the uniformity of the pore-size distribution.

\subsection{Method of lines}

The MOL technique is a popular approach for variable step size, variable-order temporal integration $[20,21]$. It is a recognized decoupling of the temporal and spatial approximations of a given solution to a system of partial differential algebraic equations. In the MOL approach, the temporal integration can be handled by sophisticated and mature algorithms and codes can be developed to solve systems of ODE or DAE aspects of the problems $[22,23]$. As a result, peoples are inspiring with the MOL technique.

To obtain the numerical solution of problems relating flow and transport in porous media, including RE, lately the MOL has become an attractive alternative approach than traditional approach [3, 17, 23, 24, 25]. For solving RE, the MOL technique has been demonstrated to be considerably more efficient than standard fixed time-step or fixed order empirically adaptive approaches [17]. Finite difference and mixed finite element spatial discretization approaches $[25,26]$, the issues involving the solution of the resulting system of algebraic equations $[26,27]$ are aspects of multidimensional and heterogeneous systems which have been investigated. With such developments, temporal integration of RE is considered relatively mature.

MOL approaches for RE require a formulation such as (2.1), which is a single equation in one unknown and is independent of the particular method of discretization in space. Mixed forms of RE, simultaneously advance $\psi$ and $\theta$ in time with a standard, low-order time integration method (e.g., fully implicit or Crank-Nicolson methods) so as to conserve mass balance. It is formally one equation in two unknowns and as such cannot be given to an ODE solver. Therefore, mixed methods typically use first-order scheme to advance $\theta$ and achieve mass balance $[6,28]$. The MOL will allow higher-order integration when based upon a high-order ODE solver.

A MOL technique based on reduction of the original equation to a set of explicit ODEs would generally require rearranging $(2.3)$ to the subsequent form:

$$
\frac{\partial \psi}{\partial t}=\frac{1}{c(\psi)+S_{S} S_{a}(\psi)} \frac{\partial}{\partial z}\left[K(\psi)\left(\frac{\partial \psi}{\partial z}+1\right)\right],
$$

which yields an explicit set of ODEs after the approximation of spatial derivatives. This approach is the clear one; on the other hand, the preliminary work found it to be an ineffective and relatively expensive approach for computing solutions to RE [17]. The difficulty with the technique is that $\mathrm{S}_{\mathrm{a}}(\psi)$ can become very small for cases when saturated conditions enlarge and fluid compressibility is small. We present here a spatial discretised DAE approach which is not divide by $S_{a}(\psi)$. Popular methods based on $(2.1)$ use this technique $[11,29]$ but are restricted to low-order methods in time.

\subsection{Spatial approximation}

The finite difference discretized RE with respect to the spatial dimension $z$, where $z \in[0, Z] \$$ and consider a uniform spatial discretization comprised of $N-1$ intervals of length $\Delta \mathrm{z}$, with $\Delta \mathrm{z}=\mathrm{Z} /(N-1)$, and $z_{i}=(i-1) \Delta z$ for $1 \leq i \leq N$.

The spatial operator :

$$
\mathrm{O}_{\mathrm{s}}(\psi)=\frac{\partial}{\partial \mathrm{z}}\left[\mathrm{K}(\psi)\left(\frac{\partial \psi}{\partial \mathrm{z}}+1\right)\right]
$$

is approximated at $\mathrm{z}=\mathrm{z}_{\mathrm{i}}$ for $1<\mathrm{i}<\mathrm{N}$ by 


$$
\begin{gathered}
O_{s i}(\psi)=\frac{\left(K \frac{\partial \psi}{\partial z}\right)_{i+1 / 2}-\left(K \frac{\partial \psi}{\partial z}\right)_{i-1 / 2}}{\Delta z}+\frac{K_{i+1 / 2}-K_{i-1 / 2}}{\Delta z} \\
=\frac{K_{i+1 / 2} \frac{\psi_{i+1}-\psi_{i}}{\Delta z}}{\Delta z}-\frac{K_{i-1 / 2} \frac{\psi_{i}-\psi_{i-1}}{\Delta z}}{\Delta z}+\frac{K_{i+1 / 2}-K_{i-1 / 2}}{\Delta z} \\
=\frac{1}{\Delta z^{2}}\left[K_{i-1 / 2} \psi_{i-1}-\left(K_{i-1 / 2}+K_{i+1 / 2}\right) \psi_{i}+K_{i-1 / 2} \psi_{i+1}\right]+\frac{1}{\Delta z}\left[K_{i+1 / 2}-K_{i-1 / 2}\right] \\
=r\left[\left(K_{i}+K_{i-1}\right) \psi_{i-1}-\left(K_{i-1}+2 K_{i}+K_{i+1}\right) \psi_{i}+\left(K_{i}+K_{i+1}\right) \psi_{i+1}\right]+\frac{1}{2 \Delta z}\left[K_{i+1}-K_{i-1}\right],
\end{gathered}
$$

where $r=\frac{1}{2 \Delta \mathrm{z}^{2}}$ and $N$ is the total number of spatial nodes in the solution, $\psi_{\mathrm{i}}$ is the approximation to $\psi\left(\mathrm{z}_{\mathrm{i}}\right)$, $\mathrm{K}_{\mathrm{i}}=\mathrm{K}\left(\psi_{\mathrm{i}}\right)$ and

$$
\begin{aligned}
K_{i+1 / 2} & =\frac{1}{2}\left[K\left(\psi_{i+1}\right)+K\left(\psi_{i}\right)\right], \\
K_{i-1 / 2} & =\frac{1}{2}\left[K\left(\psi_{i}\right)+K\left(\psi_{i-1}\right)\right],
\end{aligned}
$$

The DAE system that we solve a system of $N-2$ differential equations for the $N-2$ unknown functions of $\psi_{i}(t)$, subject to the boundary conditions $\psi_{1}$ and $\psi_{N}$. The ith equation is:

$$
A\left(\psi_{i}\right) \frac{d \psi_{i}}{d t}=O_{s i}(\psi)
$$

where $O_{s i}$ is a spatial operator given by (2.14) and

$$
A\left(\psi_{i}\right)=c\left(\psi_{i}\right)+S_{s} S_{a}\left(\psi_{i}\right)
$$

\subsection{ODE solver}

Implicit ODE or DAE integrator can be applied to solve the system of equations (2.15), with a stiff solver being the most realistic selection. The time integrator packages LSODE, VODE, DASSL, DASPK (is the latest version of DASSL) are available, which are all public domain time integrators accessible through netlib (http://www.netlib.org/). All are based upon forms of the backward differentiation formulas (BDFs). LSODE and VODE are ODE solvers, while DASSL is a DAE solver. All of these solvers are well appropriate to stiff systems of differential equations of the type encountered in the MOL solution of RE.

The MOL involves discretising the spatial domain and thus replacing the PDE with a vector system of ODE, for which efficient and effective integrating packages have been developed [30, 31]. The MATLAB package has powerful vector and matrix treatment capabilities, a good set of ODE solvers, and an widespread functionality which can be used to execute the MOL [31].

MATLAB ODE solvers ode15s is used in this work as a time integration solver, which is a variable order solver based on the numerical differentiation formulas (NDFs). It uses the backward differentiation formulas (BDFs, also known as Gear's method) that are typically less efficient. In other words, it is a quasi-constant step size implementation of the NDFs in terms of backward differences. When ode45fails, or the solution is very inefficient, and/or one expect that the problem is stiff, or when solving a differential-algebraic problem, then ode15sis an suitable ODE solver [31,32,33,34] but the accuracy of ode15sis low to medium. The MATLAB ODE solver is maintained automatic time stepping by construction, that is, solver can able to decrease or increase the require time step size to achieve the convergence within a specified tolerance.

\subsection{Performance measure}

To evaluate the robustness and efficiency of the MOL approach, we used four set of spatial nodes for each of the test cases. The number of successful steps, failed attempts, function evaluations, partial derivatives, LU decompositions, solutions of linear systems aremeasured as the computational performance of ODE solver. Characteristic of the method relative to changes in the refinement level and the cells number in the coarse grid are included in the studied area. Hence CPU time was measured as a one appropriate choice of work measure. Another important parameter for assessment of accurate results in this work is the number of nodes in the grid. The accuracy for each of the simulation is evaluated by means of the root mean square error (RMSE) and mass balance (MB) calculated with respect to the surrogate exact solution. The deviation between the fine and several sets of coarse solutions are quantified using a RMSE formula. Numerical errors are tracked by using a traditional MB approach. However, preserve an excellent closing of mass in the domain even though the internal structure of the solution was different from the fine solution. So, the calculated MB also reported for each of the runs. For an efficient and reliable numerical simulation, adequate conservation of global mass is necessary but not sufficient for acceptability. A low MB error is necessary but not sufficient condition to ensure accuracy of the solution. In other words, accurate solutions guarantee small MB error. So, we evaluated model 
performance by calculating differences with an exact solution of the RE. For this purpose, the numerical solution is computed using a very fine grid. Also, we calculated the Modeling Efficiency (ME) to make surethe accuracy and efficiency of the approach. In this study, all of the numerical codes have been written by MATLAB 7.6.0 (2008a) software and executed on a Dell INSPIRON, $2.56 \mathrm{GHz}$ system.

We consider the following formula for measures of RMSE :

$$
\|E\|_{2}=\left[\frac{1}{N} \sum_{i=1}^{N}\left(\psi_{e}-\psi_{i}\right)^{2}\right]^{\frac{1}{2}}
$$

We consider $\psi_{e}$ is the base solution, which is made by dense-grid resolution of the vertical soil column, $\psi_{i}$ is the computed solution and $N$ is the total number of nodes.

Mass balance (MB) measurement for determination the ability of a scheme for mass conservation can be defined as follows:

$\mathrm{MB}=($ Total additional mass in the domain $) /$ (Total net flux into the domain),

Here the additional mass is evaluated with respect to the initial mass in the system and it is calculated by the following formula:

$$
M B\left(t^{n}\right)=\frac{\sum_{i=2}^{N-1}\left(\theta_{i}^{n}-\theta_{i}^{o}\right) \Delta z}{\sum_{j=0}^{n}\left\{-K_{N-1 / 2}^{j}\left(\frac{\psi_{N-1}^{j}-\psi_{N}^{j}}{\Delta z}+1\right)+K_{3 / 2}^{j}\left(\frac{\psi_{1}^{j}-\psi_{2}^{j}}{\Delta z}+1\right)\right\}}
$$

where $K_{N-1 / 2}^{j}=\frac{K_{N}^{j}+K_{N-1}^{j}}{2}$ and $K_{3 / 2}^{j}=\frac{K_{1}^{j}+K_{2}^{j}}{2}, n$ is the number of time steps, $\psi_{N}^{j}$ is the pressure head in the $j$ th time and $N$ th node and $\theta_{i}^{0}$ and $\theta_{i}^{n}$ are the initial and final values of moisture content in node $i$ respectively.

The following measures of analysis [35] were also used in the comparisons:

Modeling efficiency:

$$
M E=1-\sum_{i=1}^{N}\left(\frac{\psi_{i}-\psi_{*}}{\psi_{e}-\psi_{*}}\right)^{2}
$$

where $\psi_{*}$ is the mean calculated values. The ME (desired value is 1 ), is a global model performance measure which gives the ratio of the deviations with regard to the calculated solution. ME compares fluctuations in the computed solution with the exact solution.

\section{Numerical Tests}

Before stating numerical results to assure the objectives of this work, we describe three test problems on which these investigations were based, examine methods for quantifying the accuracy of the resultant solutions, and outline methods to compute the computational work required to achieve the results. A set of numerical experiments was performed, to assess the robustness of the approach, to investigate methods for improving the efficiency of solutions to RE, to evaluate the advantage of using higher-order methods in time and make a comparison of computational performance between the MATLAB ODE solver which is adaptive in time and the published results of Miller et al. [11].

\subsection{Test problems}

Aspects of the MOL solution to RE, the approach is applied to three test problems: Problem I, Problem II, and Problem III. The simulation conditions are described in Table 1, including constitutive relation properties, spatial and temporal domains, and auxiliary conditions. In Table 1, $\Delta \mathrm{t}_{\mathrm{a}}$ is the macroscale time step for adaption. Problem I has been previously examined by Miller et al. [11], Tocci et al. [17], and Rathfelder and Abriola[28]. The material properties for Problems II, and III correspond to a dune sand as reported by Kool and Parker [36], while the auxiliary conditions vary to yield a range of solution behavior.

Problem I is a standard test problem [17] and is substantially easier than the remaining test problems for various reasons. The domain for Problem I is much shorter, the media is not as uniform and saturated conditions do not develop. Besides, it is a common test problem and very useful in clearly illustrating some important aspects of the MOL technique.

Problem II is a vertical infiltration problem and has been analyzed by Tocci et al. [17], Miller et al [17], as well as Kees et al. [37]. It has constant head boundary conditions at both top and bottom boundaries and a hydrostatic equilibrium initial condition. The combination of the initial and boundary conditions along with the constitutive relationships makes it a very difficult problem to solve accurately, since the solution includes an extremely sharp front in space that moves through the domain as a function of time.

Problem III involves vertical infiltration with redistribution [37]. It has a constant head boundary condition at the bottom of the domain and a time dependent boundary condition at the top of the domain with hydrostatic equilibrium initial conditions. The time varying boundary condition yields a difficult two-front problem. 
Table 1.Soil hydraulic properties used in the test problems

\begin{tabular}{|c|c|c|c|}
\hline Variable & Problem I & Problem II & Problem III \\
\hline$\theta_{r}(-)$ & 0.102 & 0.093 & 0.093 \\
$\theta_{s}(-)$ & 0.368 & 0.301 & 0.301 \\
$\alpha\left(\mathrm{m}^{-1}\right)$ & 3.350 & 5.470 & 5.470 \\
$\mathrm{n}(-)$ & 2.000 & 4.264 & 4.264 \\
$\mathrm{~K}_{\mathrm{s}}(\mathrm{m} / \mathrm{day})$ & 7.970 & 5.040 & 5.040 \\
$\Omega(\mathrm{m})$ & {$[00.3]$} & {$[010]$} & {$[5]$} \\
$\mathrm{t}($ days $)$ & {$[00.3]$} & {$[00.25]$} & {$[0.325]$} \\
$\psi_{0}(\mathrm{~m})$ & -10.0 & $-\mathrm{z}$ & $-\mathrm{z}$ \\
$\psi_{1}(\mathrm{~m})$ & -10.0 & 0.0 & 0.0 \\
$\psi_{2}(\mathrm{~m})$ & -0.75 & 0.1 & $-10\left(1.0-1.01 e^{-t}\right)$ \\
$\Delta \mathrm{t}_{\mathrm{a}}($ days $)$ & 0.025 & 0.025 & 0.0125 \\
\hline
\end{tabular}

\section{Results And Discussion}

Each test case runs with RelTol (relative tolerance) $=1.0 \times 10^{-6}$ and $\mathrm{AbsTol}$ (absolute tolerance) $=1.0 \times 10^{-6}$. The coarse grid for each simulation contained 51 nodes. For each of the test problems, we generated temporal adaptive simulations with grids corresponding to uniform discretizations with 151,451 , and 1351 nodes. The dense grid solutions or exact solutions for all the test cases obtained on a uniform grid of 2701 nodes with the AbsTol and RelTol both set to $1.0 \times 10^{-10}$.

\subsection{Problem I}

Figures 1 are a comparison of solution profiles for uniform grids for pressure head and aqueous phase volume fraction respectively. These solutions are very similar to the one reported in the literature [11].
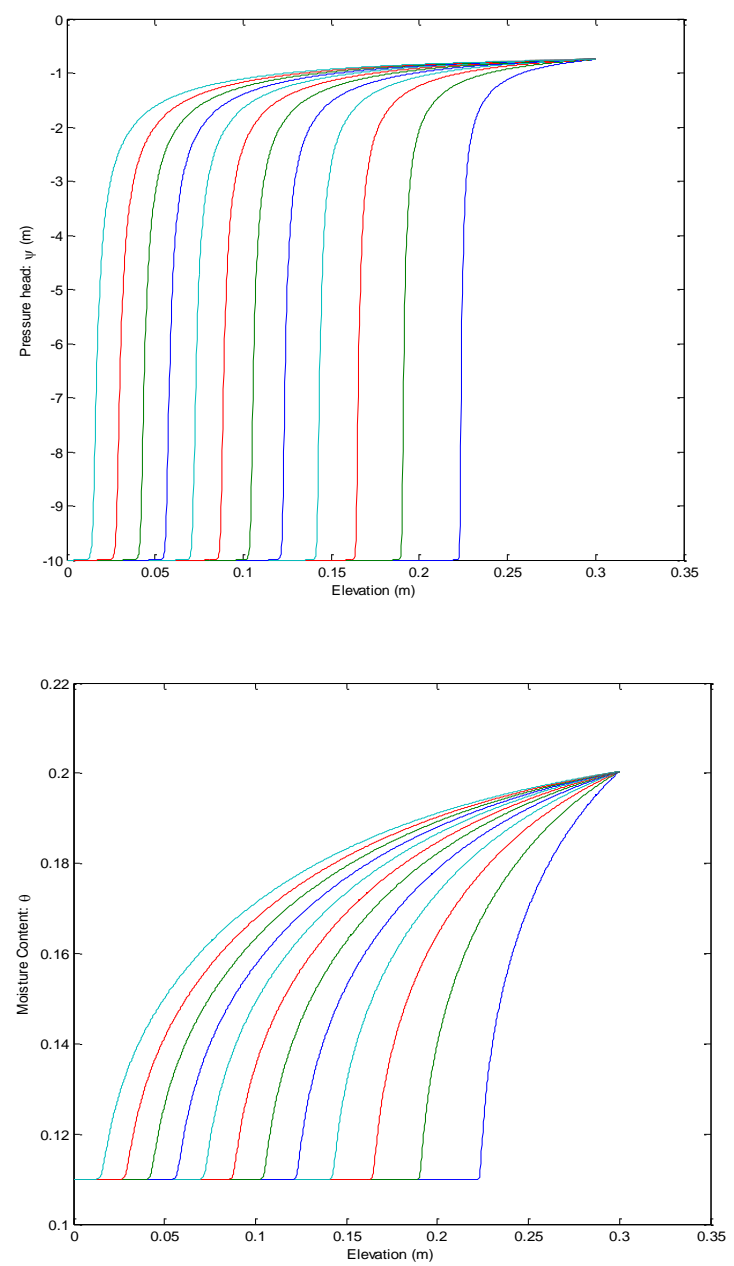
Fig. 1:Comparison of solution profiles (pressure head and aqueous phase volume fraction respectively) for uniform grids for Problem I.

A comparison of computational statistics, such as the number of steps taken, the number of function evaluations, the number of Jacobian evaluations and CPU time for the various runs are tabulated in Table 2. As well as, the number of failed attempts, the number of LU decomposition and the number of solutions of linear systems are reported but the literature [11] is out of this measurement. Results are shown in Table 2 document that we satisfied the objective of this work. The solver makes very fewer number of Jacobianevaluation than the results of Miller et al. [11] for all the runs. For example, the MATLAB solver needs only 293 Jacobian evaluation and reused it each of the 1136 times it formed and factored an iteration matrix, on the other hand, 5314 number of Jacobians calculation are needed to complete the simulation for the method used in the literature [11] in the case of 1351 nodes. All other performance of the solver used in this work is satisfactory except the CPU time on the basis of comparison with Miller et. al.. It is clearly shown that the MATLAB ODE solver is very expensive than Miller et al. approach [11] for solving such problem.

Table 2. Computational statistics of ODE solver for the Problem I

\begin{tabular}{|c|c|c|c|c|c|}
\hline & No. of nodes $\rightarrow$ & 51 & 151 & 451 & 1351 \\
\hline No. of Successful & ode15s & 1223 & 2415 & 4525 & 6857 \\
\hline Steps & Miller et.al. & 1242 & 2264 & 3732 & 5267 \\
\hline $\begin{array}{l}\text { No. of Failed } \\
\text { Attempts }\end{array}$ & $\begin{array}{c}\text { ode15s } \\
\text { Miller et.al. }\end{array}$ & $\begin{array}{c}61 \\
\text { N/A }\end{array}$ & $\begin{array}{r}124 \\
\text { N/A }\end{array}$ & $\begin{array}{l}245 \\
\text { N/A }\end{array}$ & $\begin{array}{l}414 \\
\text { N/A }\end{array}$ \\
\hline $\begin{array}{c}\text { No. of Function } \\
\text { Evaluations }\end{array}$ & $\begin{array}{c}\text { ode15s } \\
\text { Miller et.al. }\end{array}$ & $\begin{array}{l}2879 \\
3915 \\
\end{array}$ & $\begin{array}{l}9984 \\
6968 \\
\end{array}$ & $\begin{array}{l}60850 \\
11444\end{array}$ & $\begin{array}{c}410395 \\
16287\end{array}$ \\
\hline $\begin{array}{l}\text { No. of Partial } \\
\text { Derivatives }\end{array}$ & $\begin{array}{c}\text { ode15s } \\
\text { Miller et.al. }\end{array}$ & $\begin{array}{c}10 \\
1290\end{array}$ & $\begin{array}{c}33 \\
2306\end{array}$ & $\begin{array}{c}114 \\
3768\end{array}$ & $\begin{array}{c}293 \\
5314\end{array}$ \\
\hline $\begin{array}{c}\text { No. of LU } \\
\text { Decompositions }\end{array}$ & $\begin{array}{c}\text { ode15s } \\
\text { Miller et.al. }\end{array}$ & $\begin{array}{r}207 \\
\text { N/A } \\
\end{array}$ & $\begin{array}{r}386 \\
\text { N/A }\end{array}$ & $\begin{array}{r}697 \\
\text { N/A }\end{array}$ & $\begin{array}{l}1136 \\
\text { N/A }\end{array}$ \\
\hline $\begin{array}{l}\text { No. of Solutions } \\
\text { of Linear Systems }\end{array}$ & $\begin{array}{c}\text { ode15s } \\
\text { Miller et.al. }\end{array}$ & $\begin{array}{l}2378 \\
\text { N/A }\end{array}$ & $\begin{array}{l}5033 \\
\text { N/A }\end{array}$ & $\begin{array}{l}9549 \\
\text { N/A }\end{array}$ & $\begin{array}{c}14844 \\
\text { N/A }\end{array}$ \\
\hline CPU (s) & $\begin{array}{c}\text { ode15s } \\
\text { Miller et.al. }\end{array}$ & $\begin{array}{l}2.10 \\
0.85\end{array}$ & $\begin{array}{l}16.41 \\
2.52\end{array}$ & $\begin{array}{c}438.10 \\
12.83\end{array}$ & $\begin{array}{c}15020.73 \\
60.38\end{array}$ \\
\hline
\end{tabular}

The RMSE for all the vertical discretizations are presented by the Figure 2. At the end of the simulation, the highest errors for $51,151,451$, and 1351 nodes are $3.68 \mathrm{~m}, 1.31 \mathrm{~m}, 0.24 \mathrm{~m}$, and $0.02 \mathrm{~m}$ respectively shown at about $\mathrm{z}=0.013 \mathrm{~m}$. Sufficiently small errors are observed in the remaining domain of the problem. It is evident that the errors are reduced with increasing the number of nodes. RMSE, ME, amd MB errors for the four set of layers are presented in the Table 3 and acceptably small MB are found for all thediscretizations. We also analyzed residual errors because $\mathrm{MB}$ is not a sufficient criterion for comparing the relative accuracy of the models. For all the nodes ME are 1, this implies that, the solution is very close to the analytical solution. From the statistics of Table 3, it can be concluded that all runs have adequate and comparable accuracy.

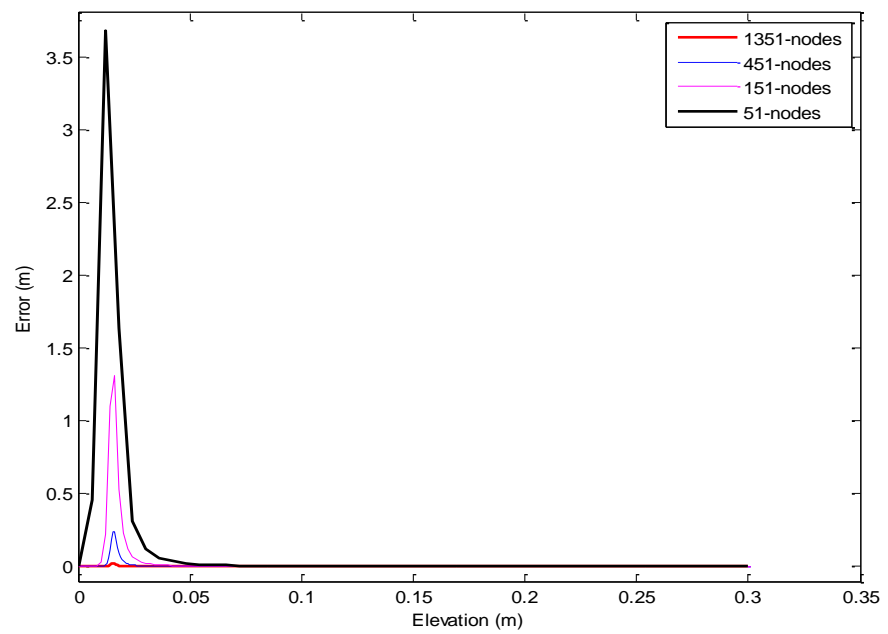

Fig. 2: Computed RMSE of all nodes for Problem I. 
Table 3.Computed RMSE, ME, and MB errors for all the vertical discretizations of Problem I.

\begin{tabular}{|c|c|c|c|c|}
\hline No. of nodes & 51 & 151 & 451 & 1351 \\
\hline RMSE & 0.5696 & 0.1487 & $2.30 \mathrm{e}-2$ & $2.30 \mathrm{e}-3$ \\
\hline ME & 1.00 & 1.00 & 1.00 & 1.00 \\
\hline MB & $4.30 \mathrm{e}-2$ & $3.00 \mathrm{e}-3$ & $4.92 \mathrm{e}-4$ & $5.48 \mathrm{e}-9$ \\
\hline
\end{tabular}

\subsection{Problem II}

A comparison of solution profiles for uniform grids for pressure head and aqueous phase volume fraction respectively are presented by the Figures 3 . The solution profiles are agreed well with the published report [11]. The computational performance of the Problem II for the various runs by the MOL approach is tabulated in the Table 4. Many fail attempts are occurred for all the cases. The CPU times column shows that the computational cost is highly increasing with increase the number of nodes. It is shown that the approach in [11] is needed approximately 10, 16, and 30 times higher numbers of Jacobians than the MOL technique by using ODE solver for the 151,451 , and 1351 nodes respectively.

It is find that from Figures 3 that the fronts are very sharp that even for 451 nodes. The maximum errors are concentrated at the front and these dominated the overall errors as they are orders of magnitude greater than the errors in the rest of the domain. The peak errors are shown at around $z=3.2 \mathrm{~m}$ and these values are $2.65 \mathrm{~m}$, $2.65 \mathrm{~m}, 2.39 \mathrm{~m}$, and $2.18 \mathrm{~m}$ for $51,151,451$, and 1351 nodes respectively.Sufficiently small errors are observed at the remaining domain. The plot (Figure 4) is shown the error behaviors of all the nodes. The evaluated RMSE, MB, and ME of all discretizations are summarized in the Table 5. All cases have given sufficiently small values, which mean that the model is robust and efficient. ME for all the cases are approaches to 1 , implies that the efficiency is very close to the analytical solution.
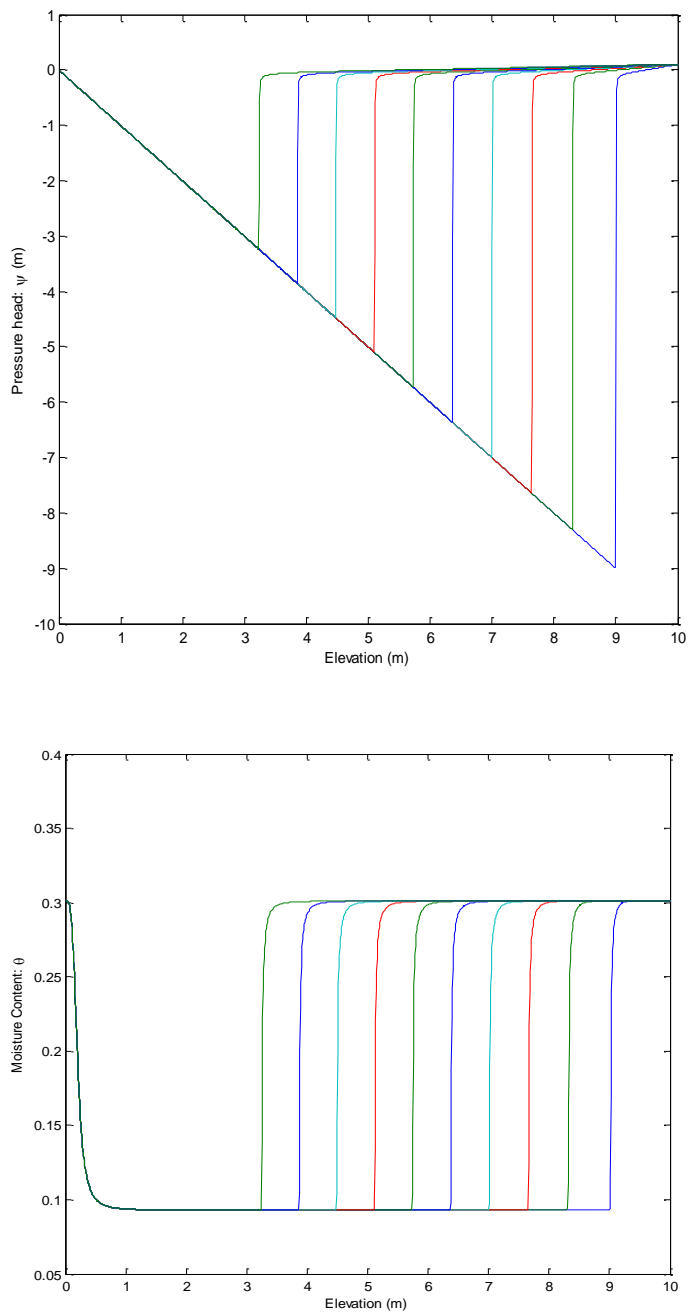
Fig. 3: Comparison of solution profiles (pressure head and aqueous phase volume fraction) for uniform grids for Problem II.

Table 4. Computational statistics of ODE solver for the Problem II

\begin{tabular}{|c|c|c|c|c|c|}
\hline & No. of nodes $\rightarrow$ & 51 & 151 & 451 & 1351 \\
\hline \multirow{2}{*}{$\begin{array}{c}\text { No. of Successful } \\
\text { Steps }\end{array}$} & ode $15 \mathrm{~s}$ & 6295 & 7560 & 21179 & 41944 \\
\hline & Miller et.al. & 3671 & 5004 & 12615 & 32989 \\
\hline \multirow{2}{*}{$\begin{array}{l}\text { No. of Failed } \\
\text { Attempts }\end{array}$} & ode15s & 2100 & 1219 & 2838 & 6965 \\
\hline & Miller et.al. & N/A & N/A & N/A & N/A \\
\hline \multirow{2}{*}{$\begin{array}{l}\text { No. of Function } \\
\text { Evaluations }\end{array}$} & ode15s & 78220 & 100352 & 422750 & $1.7512 \mathrm{e}+6$ \\
\hline & Miller et.al. & 14775 & 17153 & 45059 & 123843 \\
\hline \multirow{2}{*}{$\begin{array}{c}\text { No. of Partial } \\
\text { Derivatives }\end{array}$} & ode15s & 1230 & 554 & 837 & 1203 \\
\hline & Miller et.al. & 4128 & 5301 & 13415 & 35430 \\
\hline \multirow{2}{*}{$\begin{array}{c}\text { No. of LU } \\
\text { Decompositions }\end{array}$} & ode15s & 3071 & 1209 & 5335 & 13961 \\
\hline & Miller et.al. & N/A & N/A & N/A & N/A \\
\hline \multirow{2}{*}{$\begin{array}{l}\text { No. of Solutions } \\
\text { of Linear Systems }\end{array}$} & ode15s & 16715 & 17242 & 46086 & 127127 \\
\hline & Miller et.al. & N/A & N/A & N/A & N/A \\
\hline \multirow[t]{2}{*}{ CPU (s) } & ode15s & 55.86 & 150.74 & 3402.34 & 98559.89 \\
\hline & Miller et.al. & 1.97 & 5.67 & 44.90 & 409.54 \\
\hline
\end{tabular}

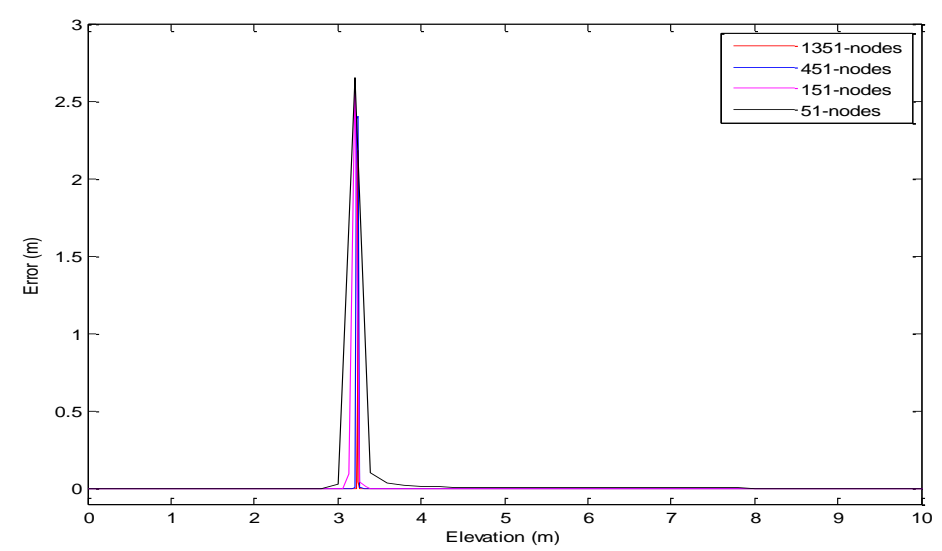

Fig. 4: Computed RMSE of all nodes for Problem II.

Table 5.Computed RMSE, ME, and MB errors for all the vertical discretizations of Problem II.

\begin{tabular}{|c|c|c|c|c|}
\hline No. of nodes & 51 & 151 & 451 & 1351 \\
\hline RMSE & 0.5696 & 0.1487 & $2.30 \mathrm{e}-2$ & $2.30 \mathrm{e}-3$ \\
\hline ME & 1.00 & 1.00 & 1.00 & 1.00 \\
\hline MB & $4.30 \mathrm{e}-2$ & $3.00 \mathrm{e}-3$ & $4.92 \mathrm{e}-4$ & $5.48 \mathrm{e}-9$ \\
\hline
\end{tabular}

\subsection{Test Problem III}

Figures 5 show a comparison of solution profiles for uniform grids for pressure head and aqueous phase volume fraction respectively and it is very similar to the published report [11]. It is obvious from the figures that there is a rapid infiltration of water from the surface is observed, followed by a period of redistribution of the water due to the dynamic boundary condition at the top of the domain.

The computational performance of the ODE solvers for the several vertical discretizations of the Problem III is reported by the Table 6. It is evident that when increase the number of layers as trice, the successful steps of ODE solvers increase by approximately 3 times for all cases whereas these are increase approximately 2.5 times for the Miller et. al. approach. Very little number of Jacobians calculations are needed for ODE solver than Miller et. al. technique.

Figure 6 shows the $L_{2}$ norm of errors and the level of accuracy are obtained clearly for all the nodes. The highest errors $5.93 \mathrm{~m}, 7.02 \mathrm{~m}, 6.19 \mathrm{~m}$, and $7.02 \mathrm{~m}$ are shown at around $z=7.5 \mathrm{~m}$ for the grids $51,151,451$, and 1351 respectively. The RMSE, ME and MB errors are summarized in the Table 7. 

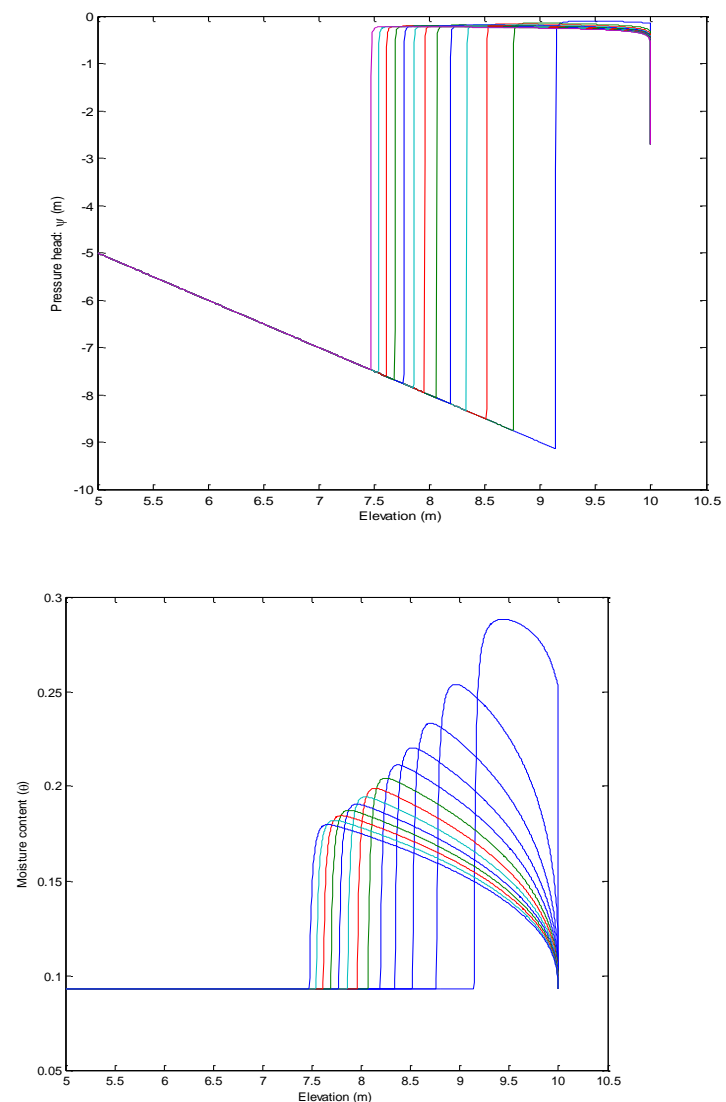

Fig. 5: Comparison of solution profiles (pressure head and aqueous phase volume fraction) for uniform grids for Problem III.

Table 6. Computational statistics of ODE solver for the Problem III

\begin{tabular}{|c|c|c|c|c|c|}
\hline & No. of nodes $\rightarrow$ & 51 & 151 & 451 & 1351 \\
\hline $\begin{array}{c}\text { No. of Successful } \\
\text { Steps }\end{array}$ & $\begin{array}{c}\text { ode15s } \\
\text { Miller et.al. }\end{array}$ & $\begin{array}{l}1937 \\
2086\end{array}$ & $\begin{array}{l}5244 \\
4363\end{array}$ & $\begin{array}{l}14674 \\
10493\end{array}$ & $\begin{array}{l}41994 \\
26477\end{array}$ \\
\hline $\begin{array}{l}\text { No. of Failed } \\
\text { Attempts }\end{array}$ & $\begin{array}{c}\text { ode15s } \\
\text { Miller et.al. }\end{array}$ & $\begin{array}{l}239 \\
\text { N/A }\end{array}$ & $\begin{array}{r}647 \\
\text { N/A }\end{array}$ & $\begin{array}{l}1840 \\
\text { N/A }\end{array}$ & $\begin{array}{l}5185 \\
\text { N/A }\end{array}$ \\
\hline $\begin{array}{l}\text { No. of Function } \\
\text { Evaluations }\end{array}$ & $\begin{array}{c}\text { ode15s } \\
\text { Miller et.al. }\end{array}$ & $\begin{array}{l}5390 \\
6996\end{array}$ & $\begin{array}{l}24582 \\
14755\end{array}$ & $\begin{array}{c}125137 \\
11444\end{array}$ & $\begin{array}{c}671949 \\
98561\end{array}$ \\
\hline $\begin{array}{l}\text { No. of Partial } \\
\text { Derivatives }\end{array}$ & $\begin{array}{c}\text { ode15s } \\
\text { Miller et.al. }\end{array}$ & $\begin{array}{c}29 \\
2236\end{array}$ & $\begin{array}{c}90 \\
4605\end{array}$ & $\begin{array}{c}209 \\
11088\end{array}$ & $\begin{array}{c}433 \\
28493\end{array}$ \\
\hline $\begin{array}{c}\text { No. of LU } \\
\text { Decompositions }\end{array}$ & $\begin{array}{c}\text { ode15s } \\
\text { Miller et.al. }\end{array}$ & $\begin{array}{l}480 \\
\text { N/A }\end{array}$ & $\begin{array}{l}1262 \\
\text { N/A }\end{array}$ & $\begin{array}{l}3404 \\
\text { N/A }\end{array}$ & $\begin{array}{l}9914 \\
\text { N/A }\end{array}$ \\
\hline $\begin{array}{l}\text { No. of Solutions } \\
\text { of Linear Systems }\end{array}$ & $\begin{array}{c}\text { ode15s } \\
\text { Miller et.al. }\end{array}$ & $\begin{array}{l}3939 \\
\text { N/A }\end{array}$ & $\begin{array}{c}11080 \\
\text { N/A }\end{array}$ & $\begin{array}{c}31080 \\
\text { N/A }\end{array}$ & $\begin{array}{c}87391 \\
\text { N/A }\end{array}$ \\
\hline CPU (s) & $\begin{array}{c}\text { ode15s } \\
\text { Miller et.al. }\end{array}$ & $\begin{array}{l}5.24 \\
1.47\end{array}$ & $\begin{array}{c}96.31 \\
5.54\end{array}$ & $\begin{array}{l}1227.76 \\
40.44\end{array}$ & $\begin{array}{c}29856.58 \\
358.10\end{array}$ \\
\hline
\end{tabular}

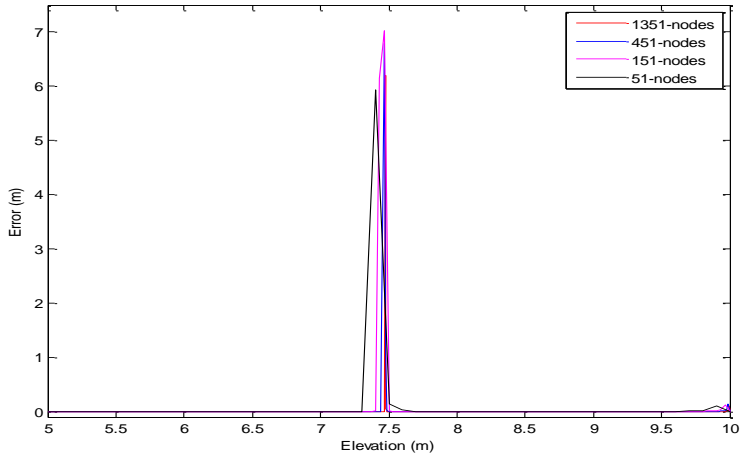

Fig. 6: Computed RMSE of all nodes for Problem III. 
Table 7.Computed RMSE, ME, and MB errors for all the vertical discretizations of Problem II.

\begin{tabular}{|c|c|c|c|c|}
\hline No. of nodes & 51 & 151 & 451 & 1351 \\
\hline RMSE & 0.8312 & 0.7600 & 0.3879 & 0.1703 \\
\hline ME & 1.00 & 1.00 & 1.00 & 1.00 \\
\hline MB & $4.9088 \mathrm{e}-5$ & 0.0 & 0.0 & 0.0 \\
\hline
\end{tabular}

\section{Conclusions}

We have formulated a spatial discretization method for a one-dimensional finite difference solution of RE. Three difficult test problems have been solved by using the MOL for a wide variety of error tolerances and spatial discretizations without difficulties. The sharp fronts were efficiently resolved under the MOL. For all the test problems, tables of computational statistics show that acceptable level of the number of function evaluations, the number of Jacobian evaluations, and the the number of steps taken are found. Therefore, the MOL framework is an efficient and robust solution of RE in one-dimension, adequate level of accuracy can be obtained with a substantial savings in computational effort and the approach implemented in this work has an excellent mass-conservation properties. Hence, the MOL approach is an attractive alternative to solve difficult sharp-front problems that arising in the RE. Many extension to this work are possible, including the development of improved error indicators or estimators, the investigation of alternative algorithmic operators for other aspects of the algorithm, and the extension to more complex multiphase problems.

\section{References}

[1] R. H. Brooks and A. T. Corey, Hydraulic properties of porous media (Hydrology Paper No.3, Civil Engineering, Colorado State University, Fort Collins, CO, 1964)

[2] M. T. van Genuchten, A Closed-form Equation for Predicting the Hydraulic Conductivity of Unsaturated Soils, Soil Sci. Soc. Am J., 44, 1980, 892-898.

[3] G. A. Williams, and C. T. Miller, An evaluation of temporally adaptive transformation approaches for solving Richards' equation, Adv. Water Resources, 1999;22(8):831-840.

[4] R. J. Hanks, and S. A. Bowers, Numerical solution of the moisture flow equation for the infiltration into layered soils, Soil. Sci. Proc., 1962:530-534.

[5] N. Romano, B. Brunone, and A. Santini, Numerical analysis of one dimensional unsaturated flow in layered soils, Adv. Water Resources, 1998;21:315-324.

[6] M. A. Celia, E. T. Bouloutas, and R. L. Zarba, A General mass-conservative numerical solution for the unsaturated flow equation, Water Resources Res., 1990;26(7):1483-1496.

[7] L. M. Abriola, and J. R. Lang, Self-adaptive finite element solution of the one dimensional unsaturated flow equation, Int. J. Numer. Methods Fluids, 1990;10: 227-246.

[8] S. H. Ju and K. J. S. Kung, Mass types, Element orders and Solution schemes for Richards' equation, Computers and Geosciences, 1997;23(2):175-187.

[9] P. C. D. Milly, A mass-conservative procedure for time-stepping in models of unsaturated flow, Adv. Water Resources, 1985;8:3236.

[10] D. Kavetski, P. Binning and S. W. Sloan, Noniterative time stepping schemes with adaptive truncation error control for the solution of Richards' equation, Water Resources Res., 2002;38(10):1211-1220.

[11] C. T. Miller, C. Abhishek and M. W. Farthing, A spatially and temporally adaptive solution of Richards' equation, Adv. Water Resources, 2005;29:525-545.

[12] D. Kavetski, P. Binning and S. W. Sloan, Adaptive backward Euler time stepping with truncation error control for numerical modelling of unsaturated fluid flow, Int. J. Numer. Meth. Eng., 2001a ;53: 1301-1322.

[13] C. Fassino and G. Manzini, Fast-secant algorithms for the non-linear Richards Equation.Communications in Numerical Methods in engineering, 1998;14: 921-930.

[14] L. Bergamaschi and M. Putti, Mixed finite elements and Newton-type linearizations for the solution of Richards' equation, Int. J. Numer.Meth. Eng., 1999;45:1025-1046.

[15] F. Lehmann and P. H. Ackerer, Comparison of iterative methods for improved solutions for fluid flow equation in partially saturated porous media, Transport in Porous Media, 1998;31:275-292.

[16] R. S. Mansell, M. Liwang, L. R. Ahuja and S. A. Bloom, Adaptive Grid Refinement in Numerical Models for Water Flow and Chemical Transport in Soil: A Review, Vadose Zone Journal, 1, 2002, 222-238.

[17] M. D. Tocci, C. T. Kelley and C. T. Miller, Accurate and economical solution of the pressure-head form of Richards' equation by the method of lines, Adv. Water Resources, 1997;20(1):1-14.

[18] R. G. Baca, J. N. Chung and D. J. Mulla, Mixed transform finite element method for solving the non-linear equation for flow in variably saturated porous media, Int. J. Numer. Meth. Fluids, 1997;24:441-455.

[19] D. Kavetski, P. Binning and S. W. Sloan, Adaptive time stepping and error control in a mass conservative numerical solution of the mixed form of Richards equation, Adv. Water Resources, 2001b;24:595-605.

[20] P. Saucez, A. VandeWouwe, and W. E. ,An adaptive method of lines solution of the Korteweg-de Vries equation, Comput. Math. Appl., 1998;35(12):13-25.

[21] W. E. Schiesser, Method of lines solution of the Korteweg-de Vries equation, Comput. Math. Appl., 1994;28(10-12):147-54.

[22] K. E. Brenan, S. L. Campbell, and L. R. Petzold,The numerical solution of initial value problems in differential-algebraic equations (Philadelphia, PA: Soc. Ind. Appl. Math., 1996).

[23] C. E. Kees and C. T. Miller, C++ implementations of numerical methods for solving differential-algebraic equations: design and optimization considerations, ACM Trans Math Software, 1999;25(4): 377-403.

[24] C. T. Miller, G. A. Williams, C. T. Kelley, and M. D. Tocci, Robust solution of Richards' equation for non uniform porous media, Water Resources Res., 1998;34:2599-2610.

[25] M. D. Tocci, C. T. Kelley, C. T. Miller, and C. E. Kees, Inexact Newton methods and the method of lines for solving Richards equation in two space dimensions, Comput. Geosci., 1999;2(4); 291-309. 
[26] M. W. Farthing, C. E. Kees, and C. T. Miller, Mixed finite element methods and higher-order temporal approximations, Adv. Water Resources, 2002;25(1); 85-101.

[27] M. W. Farthing, C. E. Kees, and C. T. Miller, Mixed finite element methods and higher order temporal approximations for variably saturated groundwater flow, Adv Water Resources, 2003;26(4);373-94.

[28] K. Rathfelder and L. M. Abriola, Mass conservative numerical solutions of the head-based Richards equation, Water Resources Res., 1994;30(9):2579-2586.

[29] P. Koorevaar, G. Menelik and C. Dirksen, Elements of soil physics. Developments in Soil Science 13(Elsevier Science Publishers B.V.,The Netherlands. 228p. 1983).

[30] W. E. Schiesser, The numerical method of lines: Integration of Partial Differential equations: ODEs, DAEs and PDEs (Academic Press, San Diego 1991).

[31] L. F. Shampineand M. W. Reichelt, The MATLAB ODE Suite Report 94-6 (Math. Dept. SMU, Dallas, 1994).

[32] L. F. Shampine and M. W. Reichelt, The MATLAB ODE Suit, SIAM J. Sci. Comput., 1997;18:1-22.

[33] L. F. Shampineand M. W. Reichelt, and J. A. Kierzenka, Solving Index-1 DAEs in MATLAB and Simulink, SIAM Review, 1999;41:538-552.

[34] L. F. Shampine, Numerical Solution of ordinary Differential Equations (Chapman and Hall, Newyork, 1994).

[35] K. Loague and R. E. Green, Statistical and graphical methods for evaluating solute transport models: Overview and application, $J$. Contam. Hydrol., 1991;7:51-73.

[36] J. B. Kool, and J. C. Parker, Development and evaluation of closed form expressions for hysteretic soil hydraulic properties, Water Resources Res., 1987;23(1):105-114.

[37] C. E. Kees and C. T. Miller, Higher order time integration methods for two-phase flow, Adv. Water Resources, 2002;25(2):15977. 